\title{
USE OF CONTACT TRACING APPS TO PROMOTE TOURISM UNDER COVID-19
}

\author{
Akira Ide \\ Kanazawa University, Japan
}

\begin{abstract}
The tourism industry has been in crisis since the explosion of the COVID-19 pandemic, and prospects for recovery remain few. Countries are showing small signs that tourists are being embraced again, with limitations being a common trend. The reason is the fear that tourists may bring COVID-19 to the area. To reduce the risk of infection and to accommodate tourists, it is necessary to show that the tourists are not infected. Several measures must be taken to avoid the dangers brought by COVID-19. Governments have developed various contact tracing applications. Indeed, information technology has proven to be especially useful. With the participation of many people, contact tracing applications will be effective in stopping infectious diseases, which in turn will help revitalize the tourism industry. This paper discusses the ideal form of contact tracing applications. The paper must have an abstract. The abstract should be self-contained and understandable by a general reader outside the context of the paper.
\end{abstract}

\section{KEYWORDS}

COVID-19, Contact Tracing App, Infection

\section{INTRODUCTION}

Since the explosion of COVID-19 cases worldwide, the tourism industry has been in crisis, and prospects for recovery appear limited at present. Despite some signs of re-acceptance of tourists, countries have tended to limit tourism activities. The reason for this is the fear that tourists may bring COVID-19 into their area.

Since the explosion of COVID-19 cases worldwide, the tourism industry has been in crisis, and prospects for recovery appear limited at present. Despite some signs of re-acceptance of tourists, countries have tended to limit tourism activities. The reason for this is the fear that tourists may bring COVID-19 into their area.

In reducing the risk of infection when accepting tourists, immigration departments must determine that the tourists are not infected. To achieve this, some islands like Singapore are considering a mechanism to conduct quarantine at airports and allow only those who have a negative polymerase chain reaction (PCR) test, which is the standard in confirming COVID-19 infections, to exit (A Singapore Government Agency Website 2020).

In the case of countries within the Schengen Agreement, tourists within the member countries are free to move around in the region. There is virtually no way to quarantine them, and they are treated with fear; locals are wary that their guests may be infected. Under these conditions, tourism promotion is impossible, and shops and hotels, especially those that have seen infections, will find it impracticable to continue operating.

Various measures can be taken to avoid the dangers of COVID-19. Information technology can be especially useful, and several governments have developed contact tracing applications. Some countries have postponed their use of these apps owing to privacy concerns(Bouffanais and Lim 2020) . However, even without the lead of a national government, these apps can be effective in deterring infections if a certain number of people use them, which will in turn help revive the tourism industry.

In this paper, we discuss the ideal form of two types of contact tracing apps. 


\section{TRENDS IN INFECTION}

The route of the spread of COVID-19 is relatively clear. Specifically, the transition from urban to rural areas can be recognized. In Italy, shortly before the lockdown of Milan and other large northern cities, a population shift to the south was observed, and then an outbreak of infection occurred in the south according to the research (Maestra,S ,et al 2020). In Japan, infections were first recorded people in urban areas and then spread to the countryside as(Japanese National Institute of Infectious Diseases 2020). This trend is not surprising. The virus, which originally appeared inland in China, was carried by travelers via airplane, subsequently spreading to other parts of the world (Singer.D 2020). Naturally, large cities with international airports are the first base of operations for those infected.

Although a small number of infected persons may be discovered in a rural area, if they are isolated for a sufficient period of time, then infection disappears, and the residents could live in peace. Meanwhile, in urban areas, the dense population contributes to the rapid spread of infection; one case can quickly lead to a cluster. Therefore, eradicating the infection in urban areas has been shown to be difficult.

Various measures have been taken in urban areas, such as banning large gatherings, but infections may be an unavoidable structural problem. Tourism, which is basically the movement of people from cities to rural areas, inevitably sends infected people to rural areas through the promotion of tourism. Consequently, the number of infected people in rural areas will not be reduced to zero. In Japan, residents in rural sites are reportedly reluctant to accept the inflow of tourists from big cities, with many cases of harassment of visitors from urban areas being recorded (NHK 2020).

For promoting tourism under such circumstances, governments must implement systems for confirming that visitors are not carriers. As described in the Introduction, the use of contact tracing apps is a helpful method. The specific usage and advantages are detailed in the following sections.

\section{EXAMPLES OF CONTACT TRACING APPS}

This section provides an overview of contact tracing apps. We will first take Japan as an example, and then examine and compare the situation in other countries. Contact tracing apps have two types: person- and location-based contact tracing.

\subsection{Person-Based Contact Tracing Apps Figures and Tables}

The Japanese contact tracing app for people is named COCOA, which is available for iPhone and Android. This app does not find out who is infected. People install and run the app, and those who may be infected will be notified. Only after they are tested can it be determined whether they are positive or not (Kurita, J, et al 2020).

Let us explain the function more concretely. Users of the app are instructed to turn on Bluetooth. The app identifies and notifies those who had close contact with infected people. First, users who feel symptoms or unwell undergo PCR testing. Second, if the result turns out to be positive, the person indicates on the app their positive status. Third, the app sends a notification to the contacts identified by the mechanism based on Bluetooth via the server. Fourth, those who receive the notification voluntarily undergo inspection and testing. If they are negative for the infection, the tracing ends there. If positive, the infected person information is entered again. Users face no penalty related to divulging their information, and doing so is at the sole discretion of the individual. In other words, the effectiveness of the app depends on the voluntary cooperation of its users.

\subsection{Location-Based Contact Tracing Apps}

The other type is location-based contact tracing. This system has been implemented by some local governments in Japan like Iwaki city (Iwaki City 2020). China, New Zealand, Singapore, South Korea, and other countries have adopted it as well, but its effectiveness has not been fully verified (Albert,M et al 2020). 
The following is a typical system in Japan. In this system, QR codes are displayed in restaurants and event venues for visitors to scan. The visitor's information is sent to a server. Later, if any of the visitors are found to be infected, they will register their information into the system. The system alerts people who had visited at the same time that an infected person visited the same site. Those who are notified will take action themselves, such as getting tested or going into voluntary quarantine. Figure1shows one kind of this system about COVID-19.

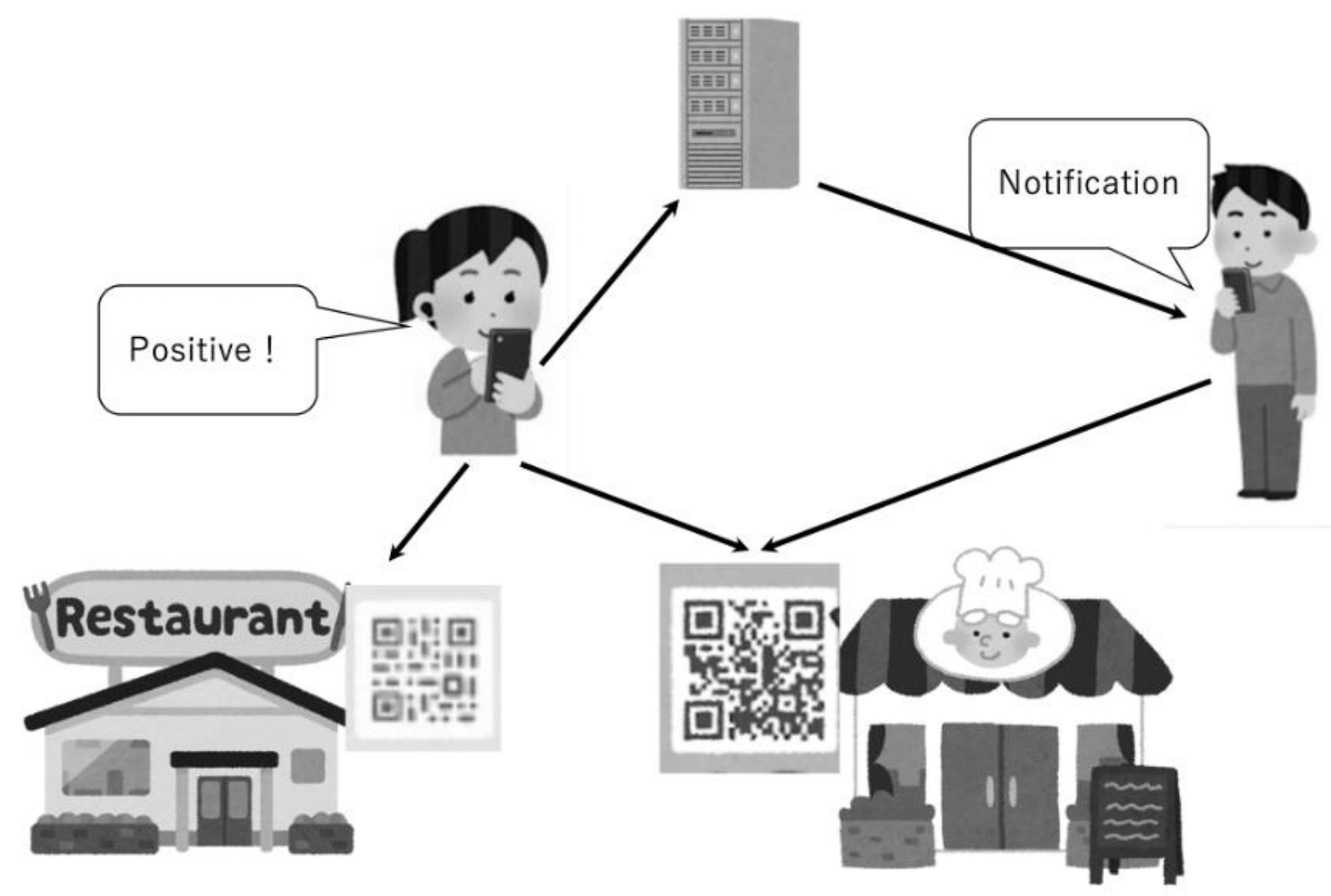

Figure 1. A typical kind of contact tracing app which based on the location

The basic structure is the same, although variations are observed across countries. In China, instead of QR codes, contactless IC chips are used. In some countries, in addition to voluntary input by the infected person, the person's information is automatically linked by the government to the personal information on the device on which the app is installed, and the government notifies the person of their test results and the people with whom the infected person may have come in contact.

\section{IDEAL TECHNOLOGY}

The above systems could be used to promote tourism. As mentioned above, these apps themselves cannot find COVID-19 positive people. They simply inform the user that a positive person had been in their vicinity within a certain time period. People confirmed to be COVID-19 positive are quarantined; they are not walking around out in the open. The problem is those infected people who are walking around not knowing they are carriers. These people are the source of the outbreak.

The appropriate response to this situation is to refuse entry of persons who may be infected. The current tracing apps, whether based on people or places, are focused on notifying the possibility of infection and calling attention. Nonetheless, using these systems helps protect the workers in restaurants, hotels, and other tourism sections. 
In the case of person-based contact tracing apps, no notification is sent if no contact with an infected person is confirmed. However, this paradigm can be shifted on its head: QR codes could be send to people who have had no contact with infected people as evidence or a security certificate that says "Until today, contact with an infected person has not been confirmed."

In location-based contact tracing apps, if a location has not had infected persons visiting it, the app could be programmed to send a separate QR code that means the person visiting it is certified as "probably safe at present." Alternatively, a QR code combined with a safety certificate could be sent and considered a guarantee. Thus, when customers enter a restaurant or event venue, they can hold the QR code over the reader, check the safety of the restaurant or event venue as well as share their own safety status, and the hosts will be more likely to accept the customers.

This system also has a public relations effect: it helps show that a location is safe. As long as an establishment's employees receive an app notification that no infected close contacts have occurred for the day, and then update the results on the app, then customers can enter with some peace of mind. In this way, the use of the app will have a synergistic effect on the safety and security of both shops and customers, which will lead to a revival of the tourism industry.

\section{PROBLEMS}

Section 4 discussed the use of an ideal contact tracing app to promote tourism. The difficulties that hinder these specifications have several different layers.

\subsection{Number of Registered Users}

Contact tracing systems will not be effective unless many people are registered. Countries such as China and Singapore that can, in a sense, "strongly" encourage their citizens to install the app can increase the number of users. In countries with liberal state systems, it is difficult to enforce the system. Various incentives are needed to increase registrations, and the role of tourism science will become increasingly important. Years of service science research have yielded knowledge on who, when, how, and what campaigns should be developed to increase the number of registrations. Measures such as effective coupon distribution should be considered.

\subsection{Compatibility Issues}

Person- and location-based contact tracing apps have different specifications in every country, and they are completely useless across countries in terms of compatibility. In Japan, the specifications of location-based contact tracing apps also differ across cities, towns, and villages; thus, even within Japan, they are no longer useful when people cross municipal boundaries. For person-based contact tracing apps, Apple and Google have presented a standardized form, but because the final product is left to each country, sharing information on infected persons across borders is not possible. This issue is explained from the perspective of personal information protection in the next section. At the system design stage, governments will have difficulty reviving international tourism without a design that will enable international information sharing.

\subsection{Personal Information Protection}

Protection of personal information is not only a matter of protecting the information and human rights of infected people. From the perspective of how infection control and tourism promotion can be achieved at the same time, it is important to find a mechanism for appropriately utilizing information related to infection.

In Japan, the management of information on infected persons is currently entrusted to local governments, and specific information on infected persons is not shared across local governments. Information shared by contact tracing apps is circulated but with personal identification information being excluded. Meanwhile, we believe that information related to location-based contact tracing apps, which is likewise not shared across municipalities, is a source of problems. Tourists can be expected to move across municipalities. If a customer 
of a restaurant is found to be infected, the customer will voluntarily enter the infection information into the app, but that information is shared only within the municipality. Given this limitation, infection data will not be available to other municipalities unless infected tourists provide such data themselves.

For location-based contact tracing apps to be effective, the information of infected persons must be automatically shared across municipalities. At the very least, if a user opts in to the terms and conditions for providing information across municipalities at the installation stage, the information should be shared with other local governments' location-based contact tracing app system of the same type. Such a mechanism for sharing information should not be limited to Japanese municipalities but should also be considered within European countries that are signatories to the Schengen Agreement. Tourists and residents are moving across borders in Switzerland and France, such as across Lake Leman and around Lugano in Switzerland. Without the sharing of information on infected people across national borders, tourists will not be able to enjoy their tourism and, in turn, the tourism industry will not be able to promote itself.

\section{CONCLUSION}

The present work is an attempt to explore the possibilities of promoting tourism amid the COVID-19 pandemic using ICT, in terms of the use of the two type of contact tracing apps. These mechanisms are intended to prevent infectious diseases, and there is room for them to be used in the future as models for the ease of personal information sharing in various types of marketing. Thus, these apps should be considered not only from the viewpoint of preventing infectious diseases but also much broadly widely, for the future development of tourism.

\section{ACKNOWLEDGEMENT}

This study was supported by JSPS KAKENHI: Grant Numbers JP18K18441, JP18K11860, JP18K12000, JP18K11886, JP20H01220.

\section{REFERENCES}

A Singapore Government Agency Website 2020, Requirements \& Process, viewed 11 September 2020, https://safetravel.ica.gov.sg/newzealand/atp/requirements-and-process

Albert M. et al 2020. Webster, C., William, R., The COVID-19-crisis and the information polity: An overview of responses and discussions in twenty-one countries from six continents. Information Polity, vol.25, no.3, pp.1-32.

Bouffanais, R\& Lim, S 2020, 'Cities - try to predict superspreading hotspots for COVID-19', Nature, vol.583, no. 7816, pp.352-355.

Iwaki City 2020, Practical use of "relief Corona general information system" has started, viewed 1 January 2021, http://honyaku.j-

server.com/LUCIWAKIC/ns/tl.cgi/http:/www.city.iwaki.lg.jp/www/contents/1593065188405/index.html

Kurita J. et al, 2020, 'Effectiveness of COCOA, a COVID - 19 contact notification application in Japan' medRxiv, viewed 1 January 2021, https://www.medrxiv.org/content/10.1101/2020.07.11.20151597v1?rss=1\%22

Maestra,S. et al. 2020, 'Epidemiological trends of COVID - 19 epidemic in Italy over March 2020: From 1000 to 100000 cases'. Journal of Medical Virology. vol.92, no. 10, pp.1956-1961.

National Institute of Infectious Diseases 2020, Current Situation of Infection, August $5^{\text {th }}$ :2020, viewed 1 January 2021, https://www.niid.go.jp/niid/en/2019-ncov-e/9806-covid19-ab5th-en.html

NHK 2020, Coronavirus sufferers face anger and prejudice, viewed 1 January 2021, https://www3.nhk.or.jp/nhkworld/en/news/backstories/1273/

Singer, D 2020. 'A new pandemic out of China: the Wuhan 2019-nCoV coronavirus syndrome'. Health Policy Technol, vol.9, no. 1, pp.1-2. 